\title{
Palmar fasciitis and polyarthritis: an uncommon but remarkable paraneoplastic syndrome
}

\author{
Hannelore Celen ${ }^{1}$ (D) B Barbara Neerinckx ${ }^{1,2}$
}

Received: 9 October 2020 / Revised: 12 December 2020 / Accepted: 14 December 2020 / Published online: 27 January 2021

(C) International League of Associations for Rheumatology (ILAR) 2021, corrected publication 2021

\section{Presentation}

An 81-year-old woman presented with a five-month history of a progressive painful swelling of the hands with flexion contractures leading to loss of hand functions (Fig. 1a). Inflammatory markers were not raised. Antinuclear antibody, anti-citrullinated peptide antibodies and rheumatoid factor were negative. Musculoskeletal ultrasound demonstrated synovitis of the extensor tendons. X-rays revealed advanced erosive osteoarthritis (Fig. 1b). Computed tomography scan exhibited widespread liver, omental and peritoneal metastases in the absence of a primary tumor. An omental biopsy showed an undifferentiated adenocarcinoma with immunohistochemical findings of a genital tract tumor. Hereby, the diagnosis of paraneoplastic palmar fasciitis and polyarthritis (PFPA) was confirmed. The tumor did not respond to chemotherapy and the musculoskeletal symptoms did not improve. The patient died four months after diagnosis.

Hannelore Celen

hannelore.celen@uzleuven.be

Division of Rheumatology, University Hospitals Leuven, Herestraat 49, 3000 Leuven, Belgium

2 Skeletal Biology and Engineering Research Centre, Department of Development and Regeneration, KU Leuven, Leuven, Belgium

\section{Discussion}

PFPA is an uncommon but remarkable paraneoplastic syndrome. Patients present with woody hands due to palmar fasciitis and synovitis of the metacarpophalangeal and proximal interphalangeal joints. Differential diagnoses include eosinophilic fasciitis, Dupuytren's contracture, systemic sclerosis and even complex regional pain syndrome (CRPS). PFPA can also mimic pseudogout, rheumatoid arthritis or remitting seronegative arthritis and symmetrical synovitis with pitting edema (RS3PE) [1, 2]. Ovarian adenocarcinomas and other female reproductive cancers are the underlying cause in more than half of the published cases, but PFPA has also been linked to other malignancies and to non-malignant medical conditions like benign cysts or tumors [1] and tuberculosis treatment [3]. Only three cases of idiopathic PFPA have been identified [4-6]. In this case, PFPA is associated with a carcinoma of unknown primary origin, which is rarely seen $[1,7]$. The effects of glucocorticoids or other immunosuppressive drugs are disappointing. Complete remission has been reported in non-malignant conditions or after complete tumor removal, but because most patients are diagnosed with advanced cancer, PFPA is often an irreversible paraneoplastic syndrome [1]. 
Fig. 1 a Progressive painful swelling of the hands and flexion contractures leading to loss of hand functions. $\mathbf{b}$ X-rays of the hands showing erosive osteoarthritis with marked deformities

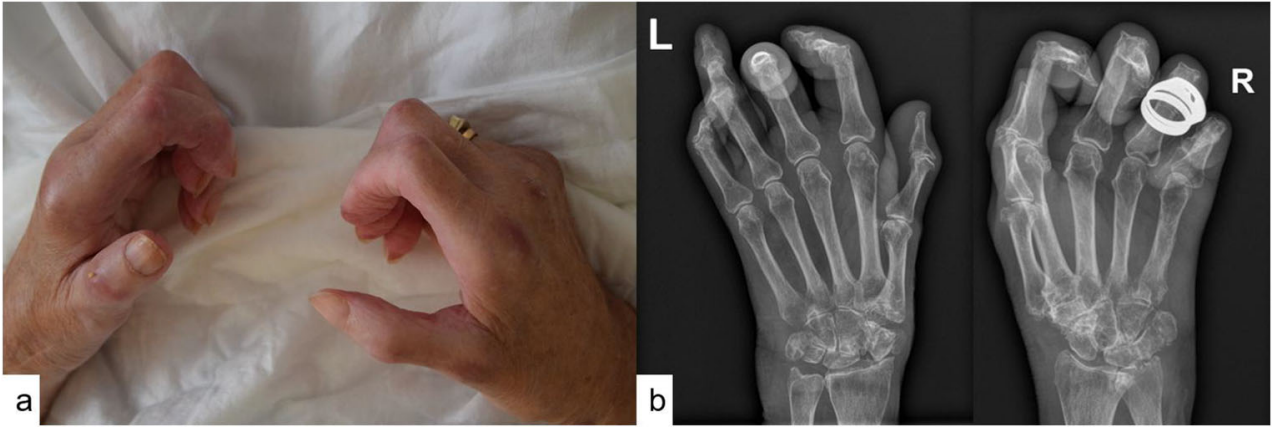

\section{Compliance with ethical standards}

\section{Disclosures None.}

Consent for publication We obtained written informed consent from the patient for publication of this case report together with any accompanying images.

\section{References}

1. Manger M, Schette G (2014) Palmar fasciitis and polyarthritis syndrome - systematic review of 100 cases. Semin Arthritis Rheum 44(1):105-111

2. Padhan P, Mishra S (2018) Palmar fasciitis and polyarthritis as a paraneoplastic syndrome. Indian J Rheumatol 13(2):129
3. Seaman JM, Goble MJ, Seaman M, Goble M, Madsen L, Steigerwald JC (1985) Fasciitis and polyarthritis during antituberculous therapy. Arthritis Rheum 28:1179-1184

4. Laszlo KS, Falanga V, Kerdel FA (1995) Idiopathic palmar fasciitis. Int J Dermatol 34:658-660

5. Sung YK, Park MH, Yoo DH (2006) Idiopathic palmar fasciitis with polyarthritis syndrome. J Korean Med Science 21:1128-1132

6. Yoshioka K, Fukumoto T, Sowa-Osako J, Tateishi C (2019) Idiopathic palmar fasciitis. BMJ case reports 12(11):e232954

7. Eekhoff EM, van der Lubbe PA, Breedveld FC (1998) Flexion contractures associated with a malignant neoplasm: a paraneoplastic syndrome? Clin Rheumatol 17(2):157-159

Publisher's note Springer Nature remains neutral with regard to jurisdictional claims in published maps and institutional affiliations. 